\title{
ON CERTAIN ASPECTS OF THE MÖBIUS RANDOMNESS PRINCIPLE
}

\author{
D. KARAGULYAN
}

\begin{abstract}
In this paper we study different aspects of the Möbius randomness principle. We rephrase the Chowla, Sarnak conjectures and the Riemann hypothesis for abstract sequences and study their relationships. We, in particular, show, that in this setting the Chowla and Sarnak conjectures do not imply the Riemann hypothesis. In the second part of the paper we also study the connection between the multiplicative and additive van der Corput criteria.
\end{abstract}

\section{INTRODUCTION}

Let $\mu$ denote the Möbius function, i.e.

$$
\mu(n)= \begin{cases}(-1)^{k} & \text { if } n=p_{1} p_{2} \cdots p_{k} \text { for distinct primes } p_{i} \\ 0 & \text { otherwise }\end{cases}
$$

It is of great importance in Number Theory because of its connection with the Riemann $\zeta$-function via the formulas

$$
\sum_{n=1}^{\infty} \frac{\mu(n)}{n^{s}}=\frac{1}{\zeta(s)}, \text { and } \sum_{n=1}^{\infty} \frac{|\mu(n)|}{n^{s}}=\frac{\zeta(s)}{\zeta(2 s)}, \text { for } \operatorname{Re}(s)>1 .
$$

We also recall the definition of the Liouville function

$$
\lambda(n)=(-1)^{\Omega(n)},
$$

where $\Omega(n)$ is the number of prime factors of $n$, counted with multiplicity. Furthermore, the estimate

$$
\left|\sum_{n=1}^{N} \mu(n)\right|=O\left(N^{\frac{1}{2}+\epsilon}\right),
$$

is equivalent to the Riemann Hypothesis [24], pp.315. We also note, that the following properties

$$
\begin{gathered}
\frac{1}{N} \sum_{n=1}^{N} \mu(n)=o(1), \text { as } N \rightarrow \infty, \\
\frac{1}{N} \sum_{n \equiv a(\bmod m)}^{N} \mu(n)=o(1), \text { as } N \rightarrow \infty,
\end{gathered}
$$

are equivalent to the prime number theorem and the Dirichlet theorem on existence of primes in arithmetic progression respectively, see e.g. [5], p. 91. 
The general Möbius pseudorandomness heuristics suggests that the sign pattern of $\mu$ behaves so randomly (or pseudorandomly) that one should expect a substantial amount of cancellation in sums that involve the sign fluctuation of the Möbius function in a nontrivial fashion, comparable to the amount that an analogous random sum would provide. There are a number of ways to make this heuristic precise. As already mentioned, the Riemann hypothesis (1.1) can be considered one such manifestation of this heuristic. Another instance of this heuristics is the following conjecture of Sarnak [16], 15].

Let $(Y, T)$ be a topological dynamical system in a compact metric space $Y$ with a continuous self map $T: Y \rightarrow Y$. The topological entropy $h(Y, T)$ of such a system is defined as

$$
h(Y, T)=\lim _{\epsilon \rightarrow 0} \lim _{n \rightarrow \infty} \frac{1}{n} \log N(\epsilon, n),
$$

where $N(\epsilon, n)$ is the largest number of $\epsilon$-separated points in $Y$ using the metric $d_{n}: Y \times Y \rightarrow \mathbb{R}^{+}$defined by

$$
d_{n}(x, y)=\max _{0 \leq i \leq n} d\left(T^{i} x, T^{i} y\right) .
$$

The existence of the limit in (1.4) is shown in [9], [12].

A sequence $f: \mathbb{Z} \rightarrow \mathbb{C}$ is said to be deterministic if it is of the form

$$
f(n)=F\left(T^{n} x\right),
$$

for all $n$ and some topological dynamical system $(Y, T)$ with zero topological entropy $h(Y, T)=0$, a base point $x \in Y$, and a continuous function $F: Y \rightarrow \mathbb{C}$. Let

$$
\epsilon=\left(\epsilon_{1}, \epsilon_{2}, \ldots\right)
$$

be a sequence of symbols from a certain alphabet $\epsilon_{k} \in\left\{a_{1}, a_{2}, \ldots, a_{m}\right\}$, and let

$$
B_{n}=\left\{\left(\epsilon_{1}, \ldots, \epsilon_{n}\right): \text { appears in } \epsilon \text { infinitely often }\right\}
$$

then the entropy of the sequence $\epsilon$ is the following limit

$$
h(\epsilon)=\lim _{n \rightarrow \infty} \frac{1}{n} \log \left|B_{n}\right| .
$$

Conjecture 1 (Sarnak, [16]). Let $f: \mathbb{N} \rightarrow \mathbb{C}$ be a deterministic sequence. Then

$$
S_{n}(T(x), f)=\frac{1}{n} \sum_{k=1}^{n} \mu(k) f(k)=o(1),
$$

as $n \rightarrow \infty$.

We note, that both $(1.2)$ and $(1.3)$ are instances of Conjecture 1. The orthogonality of the Möbius function to any sequence arising from a rotation dynamical system $(Y$ is the circle $\mathbb{T}$ and $T(x)=x+\alpha, \alpha \in \mathbb{T}$ ) follows from the following inequality of Davenport [18]

$$
\max _{\theta \in \mathbb{T}}\left|\sum_{k \leq x} \mu(k) e^{i k \theta}\right| \leq C_{A} \frac{x}{\log ^{A} x},
$$

for any $A>0$.

The conjecture is also known to be true for many other instances of zero entropy dynamical systems, see e.g. ([8], [14],[7],[18], [3]). 
Another instance of the Möbius pseudorandomness principle is the following old conjecture of Chowla.

Conjecture 2. (Chowla) Let $a_{1}<a_{2}<\cdots<a_{k}$ be natural numbers and $\epsilon_{1}, \epsilon_{2}, \ldots, \epsilon_{k} \in$ $\{1,2\}$ not all even and $k \geq 1$, then as $N \rightarrow \infty$

$$
\sum_{n=1}^{N} \mu\left(n+a_{1}\right)^{\epsilon_{1}} \mu\left(n+a_{2}\right)^{\epsilon_{1}} \cdots \mu\left(n+a_{k}\right)^{\epsilon_{k}}=o(N) .
$$

The conjecture is still open. However, there was a recent progress towards Chowla's conjecture due to K. Matomäki, M. Radziwill, T. Tao, [22], where they build on the breakthrough paper by Matomäki and Radziwill, [21]. In their paper they formulate and prove an averaged version of the Chowla conjecture.

We mention the following fundamental observation of P. Sarnak [16]

$$
\text { Chowla conjecture } \Rightarrow \text { Sarnak's conjecture. }
$$

In the proof, however, it is not used, that the Möbius function is multiplicative. It is a pure combinatorial observation. Proofs of this statement can also be found in Tao's blog [23] and in [4].

We note that all of the conjectures mentioned above are still open.

In [4] the authors rephrase the Chowla and Sarnak conjectures in abstract setting, that is, for sequences in $\{1,0,1\}^{\mathbb{N}^{*}}$ and study the relationships between these sequences and also their dynamical and ergodic properties. More specifically they consider sequences satisfying the following properties:

Definition 1. We say, that the sequence $\left\{z_{n}\right\}_{n=1}^{\infty}, z_{n} \in\{0,-1,1\}$, satisfies the Chowla property, or the property $(C h w)$ for short, if for any non-negative numbers $0=a_{0}<$ $a_{1}<a_{2}<\cdots<a_{k}$ and $\epsilon_{0}, \epsilon_{1}, \epsilon_{2}, \ldots, \epsilon_{k} \in\{1,2\}$ not all even, $k \geq 0$, we have

$$
\sum_{n=1}^{N} z_{n}^{\epsilon_{0}} z_{n+a_{2}}^{\epsilon_{2}} \cdots z_{n+a_{k}}^{\epsilon_{k}}=o(N) \text {, as } N \rightarrow \infty .
$$

Similarly inspired by Conjecture 1 we define:

Definition 2. We say, that the sequence $\left\{z_{n}\right\}_{n=1}^{\infty}, z_{n} \in\{0,-1,1\}$, satisfies the Sarnak property or the property $(S)$ for short, if

$$
\frac{1}{N} \sum_{n=1}^{N} z_{n} f(n)=o(1), \text { as } N \rightarrow \infty,
$$

for any deterministic sequence $f: \mathbb{N} \rightarrow \mathbb{C}$.

In the first section of the present paper we will continue this line of research and add the Riemann hypothesis to this list. As it was mentioned above the Riemann hypothesis is equivalent to property (1.1) of the Möbius function. Motivated by this we give the following definition:

Definition 3. We say that the sequence $\left\{z_{n}\right\}_{n=1}^{\infty}, z_{n} \in\{0,-1,1\}$, satisfies the Riemann property or the property $(R)$ for short, if for any positive $\epsilon>0$, it satisfies the 
property

$$
\sum_{n=1}^{N} z_{n}=O\left(N^{1 / 2+\epsilon}\right), \text { as } N \rightarrow \infty .
$$

As it was already mentioned above, we have $(C h w) \Rightarrow(S)$. Our goal it to investigate the relation of the property $(R)$ to the other two.

In the second part of the paper we will be interested in a consequence of one of the main tools in the study of Sarnak's conjecture, namely the following result proved by Katai [20] and Bourgain-Sarnak-Ziegler [8]:

Theorem 4 ([8], [20]). Let $\left(a_{n}\right)_{n \geq 1}$ be a sequence of bounded complex numbers, such that for any two prime numbers $p \neq q$

$$
\lim _{N \rightarrow \infty} \frac{1}{N} \sum_{n=1}^{N} a_{n p} \bar{a}_{n q}=0,
$$

then for any bounded, multiplicative function $\nu$, one has

$$
\lim _{N \rightarrow \infty} \frac{1}{N} \sum_{n=1}^{N} \nu(n) a_{n}=0 .
$$

We want to mention, that the criterion originally was formulated more quantitatively, but here we will use only the present form.

We note that the Bourgain-Sarnak-Ziegler-Katai criterion is of great importance in the study of Sarnak's conjecture. It allows to eliminate the Möbius function in the problem and reduce it to the study of intrinsic properties of the underlying dynamical system, i.e. to the study of relationship between different prime powers of the dynamical system, where one can use methods from the joining theory, the spectral theory, etc.

One can draw a simple corollary from this criterion, more specifically: if we take $\nu(n)=1$, for all $n \in \mathbb{N}$, then the condition (1.11) will imply

$$
\lim _{N \rightarrow \infty} \frac{1}{N} \sum_{n=1}^{N} a_{n}=0 .
$$

From this we get the following criterion for equidistribution of sequences. If for some sequence $\left\{b_{n}\right\}_{n=1}^{\infty}$ all the differences $\left\{\left(b_{n p}-b_{n q}\right)\right\}_{n \geq 1}$ are equidistributed modulo 1 , then so is the sequence $\left\{b_{n}\right\}_{n=1}^{\infty}$. Indeed, if for all pairs $(p, q)$ the sequence $\left\{\left(b_{n p}-b_{n q}\right)\right\}_{n=1}^{\infty}$ is equidistributed, then according to Weyl's criterion for any $k \in \mathbb{Z}$ we will have

$$
\lim _{N \rightarrow \infty} \frac{1}{N} \sum_{n=1}^{N} e^{i k\left(b_{n p}-b_{n q}\right)}=0,
$$

which is equivalent to the condition 1.10 for the sequence $c_{n}=e^{i k b_{n}}$. Therefore it will imply (1.12), which in its turn will yield the equidistribution of the sequence $\left\{b_{n}\right\}_{n=1}^{\infty}$. This criterion is also known as the multiplicative van der Corput criterion.

In the second part of the present paper we will be interested in the comparison of this criterion with the classical van der Corput criterion. 


\section{Comparison of Properties}

In this chapter we want to study the combinatorial relation between the sequences satisfying the properties $(C h w),(S)$ and $(R)$. Recall that for the Möbius function the fulfillment of the properties $(R),(C h w)$ and $(S)$ are equivalent to the Riemann hypothesis, the Chowla conjecture and the Sarnak conjecture respectively.

Consider the following two sets

$$
\left\{0=a_{0}<a_{1}<a_{2}<\cdots<a_{k}\right\} \text { and }\left\{\epsilon_{0}, \epsilon_{1}, \epsilon_{2}, \ldots, \epsilon_{k}\right\},
$$

where $k \geq 0$ and $a_{k} \in \mathbb{N}, \epsilon_{k} \in\{1,2\}$. We will briefly write this pair as $(a, \epsilon)$. We will call a pair of multi-indices $(a, \epsilon)$ admissible, if $\epsilon_{r}=1$ for at least one $0 \leq r \leq k$. For a pair $(a, \epsilon)$ of multi-indices and a sequence of real numbers $\left\{z_{k}\right\}_{k=1}^{\infty}$, we introduce the following notation

$$
z_{n}^{(a, \epsilon)}=z_{n}^{\epsilon_{0}} \cdots z_{n+a_{k}}^{\epsilon_{k}} .
$$

As we have already mention in the introduction, the Chowla property implies the Sarnak property. A proof of this implication can be found in Tao's blog [23]. We recall the basic inequality from his blog, which implies Sarnak's conjecture.

Proposition 1. Assume that the sequence $\left\{z_{n}\right\}_{n=1}^{\infty}$ satisfies the Chowla property. Then for any $m \geq 1$, any $\epsilon>0$ and any coefficients $c_{1}, \ldots, c_{m}$, such that $\left|c_{j}\right| \leq 1$, for $1 \leq j \leq m$, we have

$$
\mathbf{P}\left(\left|\frac{1}{m} \sum_{i=1}^{m} c_{i} z_{n+i}\right| \geq \epsilon\right) \leq C \exp \left(-\epsilon^{2} m / C\right)+o_{x \rightarrow \infty ; m, \epsilon}(1),
$$

where $C$ is an absolute constant and $o_{x \rightarrow \infty ; m, \epsilon}(1)$ goes to zero as $x \rightarrow \infty$ for fixed $m, \epsilon$ (uniformly in the choice of coefficients $c_{1}, \ldots, c_{m}$ ), and $n$ is uniformly distributed on $[1, x]$.

First we note, that

$$
(R) \nRightarrow(S) .
$$

Observe that from this and (1.7) it will automatically follow, that

$$
(R) \nRightarrow(C h w) .
$$

As an example to verify this, one can consider the Rudin-Shapiro sequence

$$
\begin{gathered}
a_{n}=\sum_{i=0}^{k-1} \varepsilon_{i} \varepsilon_{i+1}, \\
b_{n}=(-1)^{a_{n}}
\end{gathered}
$$

where $\left\{\varepsilon_{i}\right\}_{i=0}^{k}$ are the digits in the binary expansion of $n$. Thus, $a_{n}$ counts the number of (possibly overlapping) occurrences of the sub-string 11 in the binary expansion of $n$, and $b_{n}$ is +1 , if $a_{n}$ is even and -1 , if $a_{n}$ is odd. It is known, that the Rudin-Shapiro sequence is of zero entropy.

We now recall the following inequality for partial sums of the Rudin-Shapiro sequence. Let

$$
s_{n}=\sum_{k=0}^{n} b_{k}
$$


The following inequality is classical

$$
\sqrt{\frac{3 n}{5}}<s_{n}<\sqrt{6 n}, \text { for } n \geq 1
$$

for a proof see e.g. [10]. It follows from (2.2), that

$$
\frac{s_{n}}{n^{1 / 2+\epsilon}}<\sqrt{\frac{6}{n^{\epsilon}}}, \text { for } \epsilon>0
$$

which implies the property $(R)$. But since the entropy of the sequence is zero, then it does not satisfy the property $(S)$ since it correlates with itself.

We now construct a large class of sequences, which satisfy the property $(C h w)$ and hence the property $(S)$.

Proposition 2. Let $\left\{X_{n}\right\}_{n=1}^{\infty}$ be a sequence of independent random variables taking values in the set $\{-1,0,1\}$, with $\mathrm{E}\left[X_{n}\right] \rightarrow 0$, as $n \rightarrow \infty$. Then the sequence $\left\{X_{n}(\omega)\right\}_{n=1}^{\infty}$ satisfies the properties $(C h w)$ and $(S)$ for almost all $\omega \in \Omega$.

Proof. Recall the following form of the strong law of large numbers, see e.g. ([17], Theorem 2.3.10). Let $Y_{1}, Y_{2}, \ldots$ be a sequence of independent random variables, with

$$
\sum_{k=1}^{\infty} \frac{\operatorname{Var} Y_{k}}{k^{2}}<\infty
$$

then

$$
\frac{Y_{1}+Y_{2}+\cdots+Y_{n}}{n}-\mathrm{E}\left[\frac{Y_{1}+Y_{2}+\cdots+Y_{n}}{n}\right] \rightarrow 0
$$

almost surely. Let $(a, \epsilon)$ be an admissible pair of indices, then

$$
\sum_{n=1}^{N} X_{n}^{(a, \epsilon)}=\sum_{s=0}^{a_{k}} \sum_{n \equiv s\left(\bmod \left(a_{k}+1\right)\right) ; n \leq N} X_{n}^{(a, \epsilon)} .
$$

Consider the sum

$$
\sum_{n \equiv s\left(\bmod \left(a_{k}+1\right)\right) ; n \leq N} X_{n}^{(a, \epsilon)}=\sum_{n \equiv s\left(\bmod \left(a_{k}+1\right)\right) ; n \leq N} X_{n}^{\epsilon_{0}} X_{n+a_{1}}^{\epsilon_{1}} \cdots X_{n+a_{k}}^{\epsilon_{k}} .
$$

Note, that the sequence $\left\{X_{n}^{(a, \epsilon)}\right\}_{n \equiv s}\left(\bmod \left(a_{k}+1\right)\right)$ is a sequence of independent random variables for fixed $a_{k}$ and $0 \leq s \leq a_{k}$, since $\epsilon_{r}=1$ for at least some $0 \leq r \leq k$ and the two blocks of indices

$$
\left[n, n+a_{k}\right] \text { and }\left[m, m+a_{k}\right]
$$

are disjoint, for any two $n, m \equiv s\left(\bmod \left(a_{k}+1\right)\right), n \neq m$. For this sequence we now verify the condition 2.3 . We have, that

$$
\left|X_{n}^{(a, \epsilon)}\right| \leq 1
$$

Therefore

$$
\operatorname{Var}\left[X_{n}^{(a, \epsilon)}\right] \leq 1-\left(\mathrm{E}\left[X_{n+a_{1}}^{\epsilon_{1}} \cdots X_{n+a_{k}}^{\epsilon_{k}}\right]\right)^{2} \leq 1
$$


So the property $(2.3)$ is fulfilled. It remains to show that the second term in $(2.4)$ is converging to zero. For this, note that

$$
\mathrm{E}\left[X_{n}^{(a, \epsilon)}\right]=\mathrm{E}\left[X_{n}^{\epsilon_{0}} X_{n+a_{1}}^{\epsilon_{1}} \cdots Y_{n+a_{k}}^{\epsilon_{k}}\right]=\mathrm{E}\left[X_{n}^{\epsilon_{0}}\right] \mathrm{E}\left[X_{n+a_{1}}^{\epsilon_{1}}\right] \cdots \mathrm{E}\left[Y_{n+a_{k}}^{\epsilon_{k}}\right] \rightarrow 0
$$

as $\mathrm{E}\left[X_{n+a_{r}}^{\epsilon_{r}}\right]=\mathrm{E}\left[X_{n+a_{r}}\right] \rightarrow 0$, when $n \rightarrow \infty$. Therefore, from the general law of large number mentioned above, for the sum (2.5) we will almost surely have

$$
\lim _{N \rightarrow \infty} \frac{1}{N} \sum_{n \equiv s\left(\bmod \left(a_{k}+1\right)\right) ; n \leq N} X_{n}^{(a, \epsilon)}=0 .
$$

This finishes the proof of Proposition 2.

As a corollary from this proposition we get another proof of Theorem 2.10 (Main result 4) in [2]. The authors introduce a random model of the Möbius function defined as follows

$$
\mu_{\mathrm{rand}}(n)= \begin{cases}\epsilon_{n} & \text { if } n=p_{1} p_{2} \cdots p_{k} \text { for distinct primes } p_{k} \\ 0 & \text { otherwise }\end{cases}
$$

where $\left\{\epsilon_{n}\right\}_{n=1}^{\infty}$ is a sequence of independent and identically distributed random variables

$$
\mathrm{P}\left(\epsilon_{n}=1\right)=\frac{1}{2} \text { and } \mathrm{P}\left(\epsilon_{n}=-1\right)=\frac{1}{2} .
$$

In [2] the authors show, that the sequence

$$
\left\{\mu_{\text {rand }}(n)\right\}_{n=1}^{\infty}
$$

satisfies the property $(S)$ almost surely. Observe that if we take $X_{n}$ identically equal to 0 in Proposition 2, for square-free numbers $n$, i.e. when $n=p_{1} p_{2} \cdots p_{k}$ for distinct primes $p_{i}$, then the theorem stated above will automatically follow.

Theorem 5. The $(C h w)$ and $(S)$ properties do not imply the property $(R)$.

Proof. It is enough to construct a sequence, which satisfies the Chowla property but fails the property $(R)$. Define a sequence of independent random variables $\left\{X_{k}\right\}_{k=1}^{\infty}$ as follows

and

$$
\mathrm{P}\left(\mathrm{X}_{\mathrm{k}}=1\right)=\frac{1+\frac{1}{\log (\mathrm{k}+1)}}{2}
$$

$$
\mathrm{P}\left(\mathrm{X}_{\mathrm{k}}=-1\right)=\frac{1-\frac{1}{\log (\mathrm{k}+1)}}{2} .
$$

We are going to show, that this sequence satisfies the conditions of the theorem almost surely. First for the expected values of $X_{k}$

For the variance we have

$$
\mathrm{E}\left[X_{k}\right]=\frac{1+\frac{1}{\log (k+1)}}{2}-\frac{1-\frac{1}{\log (k+1)}}{2}=\frac{1}{\log (k+1)} .
$$

$$
\operatorname{Var}\left[X_{k}\right]=\mathrm{E}\left[X_{k}^{2}\right]-\left(\mathrm{E}\left[X_{k}\right]\right)^{2}=1-\frac{1}{\log ^{2}(k+1)}
$$

Denote

$$
S_{n}=X_{1}+X_{2}+\cdots+X_{n}
$$


Let $0<\epsilon<1 / 2$.

$$
\mathrm{E}\left[\frac{S_{n}}{n^{1-\epsilon}}\right]=\frac{1}{n^{1-\epsilon}} \sum_{k=1}^{n} \mathrm{E}\left[X_{k}\right]=\frac{1}{n^{1-\epsilon}} \sum_{k=1}^{n} \frac{1}{\log (k+1)} .
$$

But

$$
\frac{1}{n^{1-\epsilon}} \sum_{k=1}^{n} \frac{1}{\log (k+1)} \geq \frac{1}{n^{1-\epsilon}} \frac{n}{\log (n+1)}=\frac{n^{\epsilon}}{\log (n+1)}
$$

and the last expression tends to infinity, when $n \rightarrow \infty$. Hence

$$
\mathrm{E}\left[\frac{S_{n}}{n^{1-\epsilon}}\right] \rightarrow \infty, \text { as } n \rightarrow \infty
$$

We now compute the variance of $S_{n} / n^{1-\epsilon}$ :

$$
\operatorname{Var}\left[\frac{S_{n}}{n^{1-\epsilon}}\right]=\frac{1}{n^{2-2 \epsilon}} \operatorname{Var}\left[S_{n}\right]
$$

Since the random variables $X_{1}, X_{2}, \ldots$ are independent, then

$$
\begin{aligned}
\frac{1}{n^{2-2 \epsilon}} \operatorname{Var}\left[S_{n}\right] & =\frac{1}{n^{2-2 \epsilon}} \sum_{k=1}^{n} \operatorname{Var}\left[X_{k}\right] \\
& =\frac{1}{n^{2-2 \epsilon}} \sum_{k=1}^{n}\left(1-\frac{1}{\log ^{2}(k+1)}\right) \\
& \leq \frac{1}{n^{2-2 \epsilon}} n=\frac{1}{n^{1-2 \epsilon}} .
\end{aligned}
$$

Therefore, if $\epsilon<1 / 2$, then

$$
\operatorname{Var}\left[\frac{S_{n}}{n^{1-2 \epsilon}}\right] \rightarrow 0
$$

as $n \rightarrow \infty$. This means, that the sequence of random variables $S_{n} / n^{1-\epsilon}$ has high concentration around the mean. Therefore from Chebyshev's inequality, for $t>0$ we have

or

$$
\mathrm{P}\left(\left|\frac{S_{n}}{n^{1-\epsilon}}-\mathrm{E}\left[\frac{S_{n}}{n^{1-\epsilon}}\right]\right| \geq t\right) \leq \frac{\operatorname{Var}\left[\frac{S_{n}}{n^{1-\epsilon}}\right]}{t^{2}}
$$

$$
\mathrm{P}\left(\left|\frac{S_{n}}{n^{1-\epsilon}}-\mathrm{E}\left[\frac{S_{n}}{n^{1-\epsilon}}\right]\right| \geq t\right) \leq \frac{1}{n^{1-2 \epsilon} t^{2}} .
$$

Therefore, if we put $n^{2}$ instead of $n$ in the above inequality

$$
\mathrm{P}\left(\left|\frac{S_{n^{2}}}{n^{2(1-\epsilon)}}-\mathrm{E}\left[\frac{S_{n^{2}}}{n^{2-2 \epsilon}}\right]\right| \geq t\right) \leq \frac{1}{n^{2(1-2 \epsilon)} t^{2}}=\frac{1}{n^{2-4 \epsilon} t^{2}} .
$$

And if $\epsilon<\frac{1}{4}$, then we will have

$$
\sum_{n=1}^{\infty} \frac{1}{n^{2-4 \epsilon}}<\infty
$$

Therefore, if

$$
E_{n}=\left\{\omega:\left|\frac{S_{n^{2}}(\omega)}{n^{2-2 \epsilon}}-\mathrm{E}\left[\frac{S_{n^{2}}}{n^{2-2 \epsilon}}\right]\right| \geq t\right\}
$$


then the probability, that infinitely many of the events $E_{n}$ will occur is zero. So this means, that for almost all $\omega$ we have, that for infinitely many values of $n$, we have

$$
\left|\frac{S_{n^{2}}(\omega)}{n^{2-2 \epsilon}}-\mathrm{E}\left[\frac{S_{n^{2}}}{n^{2-2 \epsilon}}\right]\right|<t
$$

which, in view of (2.8), implies that

$$
\limsup _{n \rightarrow \infty}\left|\frac{S_{n^{2}}(\omega)}{n^{2-2 \epsilon}}\right|=\limsup _{n \rightarrow \infty}\left|\frac{S_{n}(\omega)}{n^{1-\epsilon}}\right|=\infty .
$$

Thus we have shown that the sequence $\left\{X_{k}\right\}_{k=1}^{\infty}$ fails the property $(R)$ almost surely for $\epsilon<\frac{1}{4}$.

To show the property $(C h w)$, we verify the requirements of Proposition 2, First, the random variables $\left\{X_{k}\right\}_{k=1}^{\infty}$ take values in the set $\{-1,1\}$ and second

$$
\mathrm{E}\left[X_{k}\right]=\frac{1}{\log (k+1)} \rightarrow 0
$$

as $k \rightarrow \infty$. So the property (Chw) follows from Proposition 2 .

Remark 1. We remark, that the result proved above contradicts with what is claimed in [1. There it is stated, that for the Liouville function the Chowla conjecture implies the Riemann hypothesis. However the multiplicativity property of the Liouville function is not used in the proof. But this can not be true as from the above argument it follows, that without the multiplicativity condition the Riemann hyphothesis can not be obtained from the Chowla property.

We note, that in the preceding theorem we used the property ( $C h w)$ to assure the property $(S)$. It is a natural question whether there is a sequence, which satisfies the property $(S)$, but fails the property $(C h w)$. As a simple example of such a sequence one can consider the following sequence

$$
X_{1} X_{1} X_{2} X_{2} \ldots X_{n} X_{n} \ldots,
$$

where $\left\{X_{n}\right\}_{n=1}^{\infty}$ is a sequence of i.i.d \pm 1 Bernoulli like random variables with parameter $\frac{1}{2}$. Denote this sequence by $\left\{Y_{n}\right\}_{n=1}^{\infty}$. Observe, that the two sequences

$$
X_{1} 0 X_{2} 0 \ldots
$$

and

$$
0 X_{1} 0 X_{2} 0 \ldots
$$

separately satisfy the property $(S)$ almost surely according to Proposition 2 . Therefore so does their sum, or the sequence (2.10). However, we have

$$
\sum_{n=1}^{N} Y_{n} Y_{n+1}=\sum_{n=1}^{[N / 2]} X_{n}^{2}+\sum_{n=1}^{[N / 2]} X_{n} X_{n+1}+o(N)=\left[\frac{N}{2}\right]+\sum_{n=1}^{[N / 2]} X_{n} X_{n+1}+o(N),
$$

where for the second term in the right hand side we have

$$
\lim _{N \rightarrow \infty} \frac{2}{N} \sum_{n=1}^{[N / 2]} X_{k} X_{k+1}=\mathrm{E}\left[X_{1} X_{2}\right]=\mathrm{E}\left[X_{1}\right] \mathrm{E}\left[X_{2}\right]=0 .
$$


So

$$
\frac{1}{N} \sum_{n=1}^{N} Y_{n} Y_{n+1}=\frac{1}{2}+o(N) .
$$

Thus the property $(C h w)$ does not hold. We note further, that the sequence 2.10 also satisfies the concentration inequality in (2.1). Since the two sequences mentioned above separately satisfy the property $(C h w)$, then they also satisfy the inequality (2.1). So one can show, that the sequence (2.10) also satisfies the concentration inequality 2.1 .

In [4] the authors construct an example of a sequence for which all the correlations in $(1.8)$, where $1<k \leq m$, fail to converge to zero for any fixed $m$. But the properties of higher other correlations are not clear. However, as the example constructed above shows, in order to have the concentration inequality in (2.1), which implies Sarnak's conjecture, one does not need the convergence to 0 of all of the admissible multiindices $(a, \epsilon)$ in Definition 1. Note that from the definition of the property $(S)$ it follows, that

$$
\lim _{n \rightarrow \infty} \frac{1}{N} \sum_{n=1}^{N} z_{n}=0
$$

which is 1.8 for $k=0$.

We now construct a sequence, for which the property $(C h w)$ fails for any $k \geq 1$ and admissible pair $(a, \epsilon)$, but that satisfies both of the properties $(S)$ and $(R)$.

Theorem 6. There is a sequence $\left\{z_{k}\right\}_{k=1}^{\infty}, z_{k} \in\{0,1,-1\}$, for which, for any $k \geq 1$ and for any pair

$$
\left\{0=a_{0}<a_{1}<a_{2}<\cdots<a_{k}\right\} \text { and }\left\{\epsilon_{0}, \epsilon_{1}, \epsilon_{2}, \ldots, \epsilon_{k}\right\},
$$

where not all $\epsilon_{k} s$ are 2 at a time, we have

$$
\lim _{N \rightarrow \infty} \frac{1}{N} \sum_{n=1}^{N} z_{n}^{(a, \epsilon)} \neq 0,
$$

but the properties $(S)$ and $(R)$ are fulfilled.

The idea of the proof is the following. First, for each pair $(a, \epsilon)$ of admissible multiindices, we construct a sequence of random variables for which the corresponding averages fail to converge to zero and are such, that it can be decomposed into two subsequences of random variables so that each subsequence consists of only independent random variables, then use Proposition 2 for each subsequence and then obtain the properties $(S)$ and $(R)$.

To prove Theorem 6 we first do some preliminary constructions and start by taking a sequence of independent and identically distributed random variables $\left\{X_{k}\right\}_{k \geq 1}$

$$
\mathrm{P}\left(X_{k}=1\right)=\frac{1}{2} \text { and } \mathrm{P}\left(X_{k}=-1\right)=\frac{1}{2} .
$$

We can assume, that

$$
X_{n}(t)=\operatorname{sgn}\left(\sin 2^{2 n} \pi t\right), \text { where } t \in[0,1], n \in \mathbb{N},
$$

or $\left\{X_{n}\right\}_{n \geq 1}$ are the even members of the sequence of Rademacher functions. 
Let $a=\left\{0=a_{0}<a_{1}<\cdots<a_{k}\right\}$. Denote $d=a_{k}+1$. We will distinguish two kinds of admissible pairs $(a, \epsilon)$ and respectively define two sequences of random variables. For an admissible pair $(a, \epsilon)$, for which $\epsilon_{r}=1$ for at least two different indices $r$, we define a sequence of random variables $\left\{Z_{n}\right\}_{n=1}^{\infty}$ as follows

$$
Z_{n}= \begin{cases}X_{n} & \text { for } n \in[1+2 d s, d+2 d s] \backslash\left\{a_{r}+2 d s+1\right\}, \\ \prod_{\substack{l=0, l \neq r}}^{k} X_{2 d s+a_{l}+1}^{\epsilon_{l}} & \text { for } n=a_{r}+2 d s+1, \\ 0 & \text { otherwise, }\end{cases}
$$

for $s=0,1, \ldots$ Observe, that if we had $\epsilon_{0}=\cdots=\epsilon_{r-1}=\epsilon_{r+1}=\cdots=\epsilon_{k}=2$, then the random variable defined for $n=a_{r}+2 d s+1$ above would be identically equal to 1 .

For a random variable $X$ we define a new random variable $Y$ satisfying the properties

$$
\mathrm{E}\left[X Y^{2}\right]=\frac{1}{2}
$$

and

$$
\mathrm{E}[Y]=0 .
$$

If $X_{n}=\operatorname{sgn}\left(\sin 2^{2 n} \pi t\right)$, for $n \in \mathbb{N}$, then we can define

$$
Y_{n}=\frac{\left(\operatorname{sgn}\left(\sin 2^{2 n} \pi t\right)+1\right)}{2} \operatorname{sgn}\left(\sin 2^{2 n+1} \pi t\right) .
$$

Observe, that for different values of $n$, the corresponding random variables $\left\{Y_{n}\right\}_{n=1}^{\infty}$ are also independent.

Now let $(a, \epsilon)$ be a pair, for which $\epsilon_{l}=1$ for all $l=0, \ldots, k$, except for $l=r$. By assumption $k \geq 1$. Let $h$ be any index different from $r$. Define a sequence of random variables as follows

$$
Z_{n}= \begin{cases}X_{n} & \text { for } n \notin[1+2 d s, d+2 d s] \backslash\left\{a_{h}+2 d s+1\right\}, \\ Y_{2 d s+a_{r}+1} & \text { for } n=a_{h}+2 d s+1 \\ 0 & \text { otherwise }\end{cases}
$$

for $s=0,1, \ldots$

Claim 1. Both in 2.12) and (2.13) we have

$$
\mathrm{E}\left[Z_{n}\right]=0 \text { and }\left\|Z_{n}\right\|_{\infty} \leq 1
$$

for all $n \geq 1$.

Proof. By definition $\mathrm{E}\left[X_{n}\right]=0$ and $\mathrm{E}\left[Y_{n}\right]=0$ for $n \geq 1$. So one only needs to check this when $n=a_{r}+2 d s+1$ for the sequence (2.12). But in this case we have

$$
\mathrm{E}\left[X_{2 d s+1}^{\epsilon_{0}} \cdots X_{2 a s+a_{r-1}+1}^{\epsilon_{r-1}} X_{2 a s+a_{r+1}+1}^{\epsilon_{r+1}} \cdots X_{2 a s+a_{k}+1}^{\epsilon_{k}}\right]=\mathrm{E}\left[X_{2 d s+1}^{\epsilon_{0}}\right] \cdots \mathrm{E}\left[X_{2 d s+a_{k}+1}^{\epsilon_{k}}\right]=0
$$

since by assumption at least one of the numbers $\epsilon_{0}, \epsilon_{1}, \ldots, \epsilon_{r-1}, \epsilon_{r+1} \ldots, \epsilon_{k}$ is different from 2. The uniform boundedness of the sequence $\left\{Z_{n}\right\}_{n \geq 1}$ follows from the boundedness of the random variables $X_{n}$ and $Y_{n}$. 
Claim 2. For each pair $(a, \epsilon)$ of admissible multi-indices and the corresponding sequence of random variables there exist two sequences of 0 's and 1 's, $\{\zeta(n)\}_{n=1}^{\infty}$ and $\{\nu(n)\}_{n=1}^{\infty}$, so that the two sequences below are sequences of independent random variables

$$
\left\{Z_{n} \nu(n)\right\}_{n=1}^{\infty},\left\{Z_{n} \zeta(n)\right\}_{n=1}^{\infty}
$$

and

$$
1=\zeta(n)+\nu(n), \text { for all } n \in \mathbb{N} .
$$

Proof. To see this for the sequence (2.12), it is enough to note that for any two numbers $s_{1} \neq s_{2}$ the random variables in each block $\left[1+2 d s_{1}, d+2 d s_{1}\right]$ and $[1+$ $\left.2 d s_{2}, d+2 d s_{2}\right]$ are independent and within the blocks $[1+2 d s, d+2 d s]$ all random variables are independent from each other, except for the one at $n=a_{r}+2 s d$, so we will define $\zeta(n)=1$ for $n=a_{r}+2 s d, s=0,1, \ldots$, and as $\nu(n)$ its compliment, i.e. $\nu(n)=1-\zeta(n), n \geq 1$. As for the sequence (2.13), we have that within the blocks $\left[1+2 d s_{1}, d+2 d s_{1}\right]$ all random variables, except for $X_{n}$ and $Y_{n}$ are independent. Therefore, if we define $\zeta(n)=1$ if $Z_{n}$ is not of type $Y_{n}$, and as $\nu(n)$ its compliment, i.e. $\nu(n)=1-\zeta(n)$, the requirements of the Claim 2 will follow.

Claim 3. For any admissible pair $(a, \epsilon)$ the corresponding sequence satisfies

$$
\lim _{N \rightarrow \infty} \frac{1}{N} \sum_{n=1}^{N} Z_{n}^{\epsilon_{0}} Z_{n+a_{1}}^{\epsilon_{1}} \cdots Z_{n+a_{k}}^{\epsilon_{k}} \geq C_{(a, \epsilon)}>0
$$

almost surely.

Proof. Consider the sum

$$
\sum_{n=1}^{N} Z_{n}^{\epsilon_{0}} Z_{n+a_{1}}^{\epsilon_{1}} \cdots Z_{n+a_{k}}^{\epsilon_{k}}=\sum_{n=1}^{N} Z_{n}^{(a, \epsilon)} .
$$

Both in 2.12 and (2.13), in the case of $n \notin[1+2 d s, d+2 d s]$, we have $Z_{n} \equiv 0$. Therefore, for $n \neq 1+2 d s$ where $s=0,1, \ldots$, one has

$$
Z_{n}^{(a, \epsilon)}=Z_{n}^{\epsilon_{0}} Z_{n+a_{1}}^{\epsilon_{1}} \cdots Z_{n+a_{k}}^{\epsilon_{k}}=0,
$$

as one of the two numbers $n$ and $n+a_{k}$ will be outside of these intervals. When $n=1+2 d s, s=0,1, \ldots$, for the sequence 2.12 we have

$$
\begin{aligned}
Z_{1+2 d s}^{(a, \epsilon)} & =X_{1+2 d s}^{\epsilon_{0}} \cdots\left(X_{1+2 d s}^{\epsilon_{0}} \cdots X_{2 d s+a_{r-1}}^{\epsilon_{r-1}} X_{2 d s+a_{r+1}+1}^{\epsilon_{r+1}} \cdots X_{d+2 d s}^{\epsilon_{k}}\right) \cdots X_{d+2 d s}^{\epsilon_{k}} \\
& =\left(X_{2 d s+1}^{2 \epsilon_{0}} X_{2 d s+a_{1}+1}^{2 \epsilon_{1}} \cdots X_{d+2 d s+1}^{2 \epsilon_{k}} \cdots X_{d+2 d s+1}^{2 \epsilon_{k}}\right)=1 .
\end{aligned}
$$

So

$$
\sum_{n=1}^{N} Z_{n}^{(a, \epsilon)}=\sum_{n \equiv 1(\bmod 2 d) ; n \leq N} 1,
$$

which implies 2.14 for the sequence $(2.12)$. As for the sequence $(2.13)$, we have

$$
Z_{1+2 d s}^{(a, \epsilon)}=Z_{1+2 d s}^{2} \cdots Z_{1+2 d s+a_{r}} \cdots Z_{1+2 d s+a_{k}}^{2}=X_{1+2 d s+a_{r}} Y_{1+2 d s+a_{r}}^{2} .
$$


From this it follows, that

$$
\sum_{n=1}^{N} Z_{n}^{(a, \epsilon)}=\sum_{n \equiv 1} X_{n+a_{r}} Y_{n+a_{r}}^{2} .
$$

Since the random variables in the right hand side are independent, identically distributed and $\mathrm{E}\left[X_{n+a_{r}} Y_{n+a_{r}}^{2}\right]=1 / 2$, then

$$
\lim _{N \rightarrow \infty} \frac{1}{N} \sum_{n \equiv 1} X_{n+a_{r}} Y_{n+a_{r}}^{2}=\frac{1}{4 d}
$$

almost surely. If we now set $C_{(a, \epsilon)}=(4 d)^{-1}$, then (2.14) will follow, and this will complete the proof of Claim 3 .

Proof of Theorem 6. We now turn to the construction of a sequence, which fails the property $(C h w)$ and satisfies the properties $(S)$ and $(C h w)$. Enumerate all possible admissible pairs for $k \geq 1$

$$
\left\{a_{0}=0<a_{1}<a_{2}<\cdots<a_{k}\right\} \text { and }\left\{\epsilon_{0}, \epsilon_{1}, \epsilon_{2}, \ldots, \epsilon_{k}\right\},
$$

in such a way, that any pair appears in the enumeration infinitely often. Let

$$
\left\{\left(a_{k}, \epsilon_{k}\right)\right\}_{k=1}^{\infty}
$$

be the enumeration.

We now construct blocks $\left\{A_{k}\right\}_{k=1}^{\infty}$ of random variables and sets $\left\{B_{k}\right\}_{k=1}^{\infty}$ by induction. For the first pair $\left(a_{1}, \epsilon_{1}\right)$ from 2.16 we choose a number $N_{1}$ so that

$$
\frac{1}{N_{1}} \sum_{n=1}^{N_{1}} Z_{k=1}^{\left(a_{1}, \epsilon_{1}\right)}>C_{\left(a_{1}, \epsilon_{1}\right)}>0
$$

on a set $B_{1}$ with measure $\left|B_{1}\right|>1 / 2$. The existence of such $N_{1}$ follows from Claim 3. We will define the first block $A_{1}$ to be the following finite set of random variables

$$
\left\{Z_{k}\right\}_{k=1}^{N_{1}}
$$

In the 2nd step, we consider the pair $\left(a_{2}, \epsilon_{2}\right)$ from (2.16) and construct another block of functions $A_{2}$ of $N_{2}-N_{1}$ many random variables

$$
\left\{Z_{k}\right\}_{k=N_{1}+1}^{N_{2}},
$$

where $N_{2}$ is chosen in such a way that

$$
\frac{1}{N_{2}}\left(\sum_{n=1}^{N_{1}} Z_{k=1}^{\left(a_{2}, \epsilon_{2}\right)}+\sum_{n=N_{1}+1}^{N_{2}} Z_{k=1}^{\left(a_{2}, \epsilon_{2}\right)}\right)>C_{\left(a_{2}, \epsilon_{2}\right)}>0,
$$

on a set $B_{2}$ of measure $\left|B_{2}\right|>1-1 / 4$. The existence of $N_{2}$ follows from (2.14) and the following uniform estimate

$$
\left|\frac{1}{N_{2}} \sum_{n=1}^{N_{1}} Z_{k=1}^{\left(a_{2}, \epsilon_{2}\right)}\right| \leq \frac{N_{1}}{N_{2}} \rightarrow 0, \text { as } N_{2} \rightarrow \infty .
$$


In a similar way in the $m$ th step we consider the pair $\left(a_{m}, \epsilon_{m}\right)$ and construct a block $A_{m}$ of $N_{m}-N_{m-1}$ random variables chosen in such a way, that

$$
\frac{1}{N_{m}}\left(\sum_{n=1}^{N_{1}} Z_{n=1}^{\left(a_{m}, \epsilon_{m}\right)}+\cdots+\sum_{n=N_{m-1}+1}^{N_{m}} Z_{n=1}^{\left(a_{m}, \epsilon_{m}\right)}\right)>C_{\left(a_{m}, \epsilon_{m}\right)}>0
$$

on a set $B_{m}$ of measure $\left|B_{m}\right|>1-1 / 2^{m}$. By this procedure we will construct a sequence of blocks $\left\{A_{k}\right\}_{k=1}^{\infty}$ of random variables and sets $\left\{B_{k}\right\}_{k=1}^{\infty}$. To finish the construction, we will put all the blocks of random variables constructed above together, i.e.

$$
A_{1} A_{2} \ldots A_{k} \ldots
$$

Consider the resulting sequence $\left\{Z_{k}\right\}_{k=1}^{\infty}$. We are going to show that this sequence satisfies the required properties almost surely. Since $\left|B_{k}\right|>1-1 / 2^{k}$, then we can find a set $B$ of full measure $|B|=1$, for which

$$
B=\liminf _{k \rightarrow \infty} B_{k}
$$

i.e. any point from $\omega \in B$ eventually falls into all sets $B_{k}$, for $k \geq k_{0}(\omega)$. Let us show that for any pair $(a, \epsilon)$ we almost surely have

$$
\limsup _{N \rightarrow \infty} \frac{1}{N} \sum_{n=1}^{N} Z_{n}^{(a, \epsilon)}>0 .
$$

Since in the enumeration (2.16) any pair $(a, \epsilon)$ occurs infinitely often, then there will exist a sequence $\left\{N_{k_{m}}\right\}_{m=1}^{\infty}$, for which

$$
\frac{1}{N_{k_{m}}} \sum_{n=1}^{N_{k_{m}}} Z_{n}^{(a, \epsilon)}>C_{(a, \epsilon)}
$$

on the set $B_{k_{m}}$. Therefore this estimate will eventually hold for any point in $B$.

We will now show, that the sequence $\left\{Z_{k}\right\}_{k=1}^{\infty}$ satisfies the property $(S)$. For this let us consider the two sequences

$$
\{\zeta(n)\}_{n=1}^{\infty} \text { and }\{\nu(n)\}_{n=1}^{\infty},
$$

where each of them is a union of the corresponding sequences $\zeta$ and $\nu$ for each block $B_{k}$ constructed in Claim 2, Therefore, according to Claim 2

$$
Z_{n} \nu(n)+Z_{n} \zeta(n)=Z_{n} \text {, for any } n \geq 1,
$$

and the random variables in each of the sequences below will be independent

$$
\left\{Z_{n} \nu(n)\right\}_{n=1}^{\infty} \text { and }\left\{Z_{n} \zeta(n)\right\}_{n=1}^{\infty},
$$

and from Claim 1

$$
\mathrm{E}\left[Z_{n}\right]=0, \text { for any } n \in \mathbb{N} .
$$

From this, 2.19) and Proposition 2 we get that the two sequences in 2.20) separately satisfy the property $(S)$. Therefore it is also satisfied by $\left\{Z_{n}\right\}_{n=1}^{\infty}$.

Since each sequence in (2.18) is a sequence of independent and bounded random variables, the property $(R)$ follows from the central limit theorem. 
Remark 2. In the discussion above we did not assume, that the sequence $\{z(n)\}_{n=1}^{\infty}$ is multiplicative.

Of course the assumption of multiplicativity will change the situation substantially, however we would like to note, that the sole condition of multiplicative, the uniform boundedness and even the convergence to zero of its Cesaro averages do not guaranty the fulfillment of any of the properties $(R),(S)$ and $(C h w)$.

Consider the Dirichlet character $\chi_{1}(n)$ modulo 3, i.e.

$$
\chi_{1}(n)= \begin{cases}0 & \text { if } n \equiv 0(\bmod 3), \\ 1 & \text { if } n \equiv 1(\bmod 3), \\ -1 & \text { if } n \equiv 2(\bmod 3) .\end{cases}
$$

Clearly

$$
\lim _{N \rightarrow \infty} \frac{1}{N} \sum_{n=1}^{N} \chi_{1}(n)=0
$$

but

$$
\lim _{N \rightarrow \infty} \frac{1}{N} \sum_{n=1}^{N} \chi_{1}(3 n+1)=1 .
$$

Therefore the properties $(S),(R)$ and $(C h w)$ are not satisfied. To the best of the author's knowledge there are not any known examples of nontrivial multiplicative sequences, which satisfy the property $(S)$ or $(C h w)$. However, there are nontrivial examples of multiplicative sequences which satisfy the property $(R)$. Consider

$$
f(n)= \begin{cases}\prod_{p \mid n} \epsilon_{p} & \text { if } n \text { is squarefree, } \\ 0 & \text { otherwise }\end{cases}
$$

where $\left\{\epsilon_{n}\right\}_{n=1}^{\infty}$ is as in 2.7). It is shown in [26] that this sequence satisfies the property $(R)$ almost surely. In this context it also important to mention a result of Frantzikinakis [13], which states the following: if the Liouville function $\lambda$ is ergodic, then it satisfies the Chowla conjecture. However, as is mentioned in [13], it is not even known whether this system is of positive entropy, weakly mixing, or even ergodic.

\section{COMPARISION OF CRITERIA}

We recall that the multiplicative van der Corput criterion states the following: if for some sequence $\left\{b_{n}\right\}_{n=1}^{\infty}$ we have, that for any $p, q$ prime numbers the sequence $\left\{\left(b_{n p}-b_{n q}\right)\right\}_{n=1}^{\infty}$ is uniformly distributed $\bmod 1$, then so is $\left\{b_{n}\right\}_{n=1}^{\infty}$. Therefore one gets a criterion for equidistribution for sequences, which may be interpreted as a multiplicative version of the classical van der Corput criterion. We now recall the classical van der Corput criterion.

Theorem 7. Let $\left\{a_{n}\right\}_{n=1}^{\infty}$ be a sequence of bounded real numbers such, that for any number $h \in \mathbb{N}$ all of the differences $\left\{\left(a_{n+h}-a_{n}\right)\right\}_{n=1}^{\infty}$ are uniformly distributed modulo 1. Then the sequence $\left\{a_{n}\right\}_{n=1}^{\infty}$ is also uniformly distributed modulo 1.

In this section we want to investigate the connection between the two criteria mentioned above. If, for instance, the additive van der Corput criterion yields the 
convergence of any of the differences appearing in the additive van der Corput criterion, then it may give another tool for studying Sarnak's conjecture. However, as it will be shown next, there is no connection between the criteria. However it worth mentioning the following result of Korobov and Postnikov [19].

Theorem 8. Assume, that for the sequence $\left\{a_{n}\right\}_{n=1}^{\infty}$ the differences $\left\{\left(a_{n+h}-a_{n}\right)\right\}_{n \geq 1}$ are uniformly distributed mod 1 for any $h \in \mathbb{N}$, then one also has equidistribution for the sequences $\left\{a_{n p+l}\right\}_{n \geq 1}$, for $l, p \in \mathbb{N}$.

In particular the sequence $\left\{a_{n p}\right\}_{n=1}^{\infty}$ is equidistributed for any $p \geq 1$.

We observe that it is not difficult to construct a sequence, which satisfies the multiplicative criterion, but fails the additive one. As a simple example, one can consider the sequence $\{n \theta(\bmod 1), n \in \mathbb{N}\}$ for any irrational $\theta$. We see that for any $h \in \mathbb{N}$

$$
\{(n+h) \theta\}-\{n \theta\}=(n+h) \theta+h_{1}-n \theta-h_{2}=h \theta+h_{1}-h_{2},
$$

which is apparently not equidistributed, however one can check from Weyl's criterion, that the differences $\{n p \theta\}-\{n q \theta\}=n(p-q) \theta+m, m \in \mathbb{Z}$ is equidistributed for any $p \neq q, p, q \in \mathbb{Z}$.

We now investigate the other direction of the question. We will construct an example of a uniformly distributed sequence, for which all of the van der Corput differences are equidistributed, while none of the multiplicative differences are.

To construct a counterexample we will need a few lemmas.

Lemma 1. Let $\left\{a_{n}\right\}_{n=1}^{\infty}$ be a sequence of numbers from $[0,1)$, for which the set $\{n$ : $\left.a_{n}=0\right\}$ has positive upper density. Then $\left\{a_{n}\right\}_{n=1}^{\infty}$ is not u.d. $\bmod 1$.

Proof. Let the upper Banach density of the set $\left\{n: a_{n}=0\right\}$ be $\tau$. We now consider an interval which contains the origin $\{0\}$ in its interior and whose length is strictly smaller then $\tau$. If now $\left\{a_{n}\right\}_{n=1}^{\infty}$ is uniformly distributed, then the density of the members falling inside this interval will be smaller then $\tau$. This is a contradiction.

We now enumerate all pairs of distinct prime numbers $(p, q)$, where $p>q$

$$
\left\{\left(p_{k}, q_{k}\right)\right\}_{k=1}^{\infty}
$$

in such a way, that any pair occurs in $(3.1)$ infinitely often. For each $k$ we also define two sets

$$
A_{k}=\left\{\left(p_{k} q_{k} l+1\right) p_{k}: l \in \mathbb{N}\right\}
$$

and

$$
B_{k}=\left\{\left(p_{k} q_{k} l+1\right) q_{k}: l \in \mathbb{N}\right\} .
$$

Notice, that the two sets do not intersect for the same $k$. Indeed, if

$$
\left(s p_{k} q_{k}+1\right) q_{k}=\left(l p_{k} q_{k}+1\right) p_{k}
$$

for some $s, l \in \mathbb{N}$, then

$$
p_{k} q_{k}\left(s q_{k}-l p_{k}\right)=p_{k}-q_{k}
$$

but since $p_{k}-q_{k} \neq 0$ and at least one of the two inequalities

$$
\left|p_{k}-q_{k}\right|<p_{k} \text { or }\left|p_{k}-q_{k}\right|<q_{k}
$$


holds, then clearly the equality above can not be fulfilled. For $n \in B_{k}$, i.e. for a number of the form $n=\left(p_{k} q_{k} l+1\right) p_{k}$ we define $\bar{n}$ to be the number

$$
\bar{n}=\left(p_{k} q_{k} l+1\right) q_{k}
$$

We now construct a sequence of integers $\left\{d_{k}\right\}_{k=1}^{\infty}$, where $d_{k} \in \mathbb{Z}$. The construction will proceed by induction. Together with $\left\{d_{k}\right\}_{k=1}^{\infty}$ we will also construct a sequence $\left\{N_{k}\right\}_{k=1}^{\infty}$. Let $\left\{c_{n}\right\}_{n \geq 1}$ be a monotone sequence of positive integers, for which $\left(c_{n+1}-\right.$ $\left.c_{n}\right) \rightarrow \infty$, as $n \rightarrow \infty$. For the first step of the induction $m=1$ we consider the pair $\left(p_{1}, q_{1}\right)$. We define $N_{1}=\left(p_{1} q_{1}+1\right) p_{1}$. Also set

$$
d_{n}= \begin{cases}c_{n} & \text { for } n \leq N_{1} \text { and } n \notin B_{1}, \\ c_{\bar{n}} & \text { for } n \leq N_{1} \text { and } n \in B_{1} .\end{cases}
$$

For the correctness of the above definition we first define $d_{n}$ for $n \leq N_{1}, n \notin B_{1}$ and then for other $n \leq N_{1}$. One can check, that from the definition of $d_{n}$ it follows that $a_{\left(p_{1} q_{1}+1\right) p_{1}}-a_{\left(p_{1} q_{1}+1\right) q_{1}}=0$. This concludes the first step of the induction. Now assume we have defined $d_{n}$ up to $n \leq m-1$ and we want to define it further. Consider the pair $\left(p_{m}, q_{m}\right)$. Let $l$ be the smallest integer, for which

$$
\left(p_{m} q_{m} l+1\right) q_{m}>N_{m-1},
$$

or the smallest number from the set $B_{m-1}$, which is larger then $N_{m-1}$. We define $N_{m}=\left(p_{m} q_{m}\left(N_{m-1}+l-1\right)+1\right) p_{m}$. We now define $d_{n}$ in the following way:

$$
d_{n}= \begin{cases}c_{n} & \text { for } N_{m-1}<n \leq N_{m} \text { and } n \notin B_{m}, \\ c_{\bar{n}} & \text { for } N_{m-1}<n \leq N_{m} \text { and } n \in B_{m} .\end{cases}
$$

One can see, that

$$
\frac{N_{m}}{N_{m-1}}<\left(2 p_{m} q_{m}+1\right) p_{m}
$$

This finishes the induction. We now make a few observation about the construction.

Lemma 2. For any $h \in \mathbb{N}$, let

$$
d_{n+h}-d_{n}=c_{n}^{(h)}
$$

then $c_{n}^{(h)} \in \mathbb{Z}$ and $\left|c_{n}^{(h)}\right| \rightarrow \infty$, as $n \rightarrow \infty$.

Proof. Since $c_{n} \in \mathbb{N}$, then clearly $c_{n}^{(h)} \in \mathbb{Z}$. Let

$$
d_{n+h}=c_{n_{1}} \text { and } d_{n}=c_{n_{2}} .
$$

From the construction of the sequence $\left\{d_{n}\right\}_{n=1}^{\infty}$ it follows, that if $n>N_{k}$, for some $k \geq 0$, then $n_{2}, n_{1}>N_{k}$ too. Hence, if $n_{2} \neq n_{1}$, then

$$
\left|d_{n+h}-d_{n}\right|=\left|c_{n_{1}}-c_{n_{2}}\right|>\left|c_{n_{1}}-c_{n_{1}-1}\right| \rightarrow \infty,
$$

whenever $n \rightarrow \infty$. It remains to see, that $n_{2} \neq n_{1}$ when $n$ is sufficiently large. But this can happen if only $n+h=s$ and $n=\bar{s}$, for some $s>0$, or

$$
n+h=\left(p_{m} q_{m} l+1\right) p_{m}
$$

and

$$
n=\left(p_{m} q_{m} l+1\right) q_{m},
$$


for some $m, l, p_{m}$ and $q_{m}$. But then

$$
h=\left(p_{m} q_{m} l+1\right) p_{m}-\left(p_{m} q_{m} l+1\right) q_{m}=\left(p_{m} q_{m} l+1\right)\left(p_{m}-q_{m}\right) .
$$

The right hand side achieves its minimum for $p_{m}=3$ and $q_{m}=2$. Hence

$$
h \geq 6 l+1 \text {. }
$$

But since $l \rightarrow \infty$, when $n \rightarrow \infty$, then this can not happen, so $n_{1} \neq n_{2}$, for sufficiently large $n$.

Lemma 3. For any $(p, q)$

$$
\limsup _{N \rightarrow \infty} \frac{\#\left\{n: d_{n p}-d_{n q}=0, n \leq N\right\}}{N}>0 .
$$

Proof. By construction any pair of primes $(p, q)$ occurs in the enumeration (3.1) infinitely often. Let $\left\{m_{k}\right\}_{k=1}^{\infty}$ be the occasions when one encounters the pair $(p, q)$. By construction, if $n p \in B_{m_{k}}$, then $d_{n p}-d_{n q}=0$ and

$$
\#\left\{n: N_{m_{k}}<n \leq N_{m_{k+1}}, n \in B_{m_{k}}\right\}=N_{m_{k}} .
$$

From this and $(3.2)$, we have

$$
\frac{\#\left\{n: d_{n p}-d_{n q}=0, n \leq N_{m_{k}+1}\right\}}{N_{m_{k}+1}} \geq \frac{N_{m_{k}}}{N_{m_{k}+1}}>\frac{1}{(2 p q+1) p}>0 .
$$

From this we get 3.4 .

We now recall the following theorem from the theory of uniformly distributed sequences:

Theorem 9 ([6]). Let $\left\{a_{n}\right\}_{n \geq 1}$ be a sequence of integers with $\left|a_{n}\right| \rightarrow \infty$, for $n \rightarrow \infty$, then for almost all $\theta \in[0,1)$ the sequence $\left\{a_{n} \theta\right\}_{n=1}^{\infty}$ is u.d. $\bmod 1$.

We are now ready to construct the example of a sequence, that satisfies the classical van der Corput criterion, but fails the multiplicative one. Consider the sequence $\left\{d_{n} \theta\right\}_{n=1}^{\infty}$. From Lemma 2 we have, that $\left\{\left(d_{n+h}-d_{n}\right) \theta\right\}=\left\{c_{n}^{(h)} \theta\right\}$, where $\left|c_{n}^{(h)}\right| \rightarrow \infty$, hence from Theorem 9 it follows, that $\left\{\left(d_{n+h}-d_{n}\right) \theta\right\}_{n=1}^{\infty}$ is uniformly distributed for almost all $\theta \in[0,1)$. While for any pair $(p, q)$ from Lemma 3 we have, that

$$
\limsup _{N \rightarrow \infty} \frac{\#\left\{n: d_{n p} \theta-d_{n q} \theta=0, n \leq N\right\}}{N}>0 .
$$

Hence $\left\{\left(d_{n p}-d_{n q}\right) \theta\right\}_{n=1}^{\infty}$ cannot be uniformly distributed for any $\theta \in[0,1)$ according to Lemma 1. Thus we conclude, that for almost all $\theta \in[0,1)$ the sequence $\left\{d_{n} \theta\right\}_{n=1}^{\infty}$ is of the type required.

\section{ACKNOWLEDGEMENTS}

The author would like to express his gratitude to Michael Benedicks for his guidance and valuable suggestions and also to Lilian Matthiesen and Pär Kulberg for helpful discussions. 


\section{REFERENCES}

[1] E. H. el Abdalaoui, On the Erdös flat polynomials problem, Chowla conjecture and Riemann Hypothesis, https://arxiv.org/abs/1305.4361.

[2] E. H. el Abdalaoui, M. Disertori, Spectral properties of the Möbius function and a random Möbius model, https://arxiv.org/abs/1305.4361.

[3] H. El Abdalaoui, M. Lemanczyk, T. de la Rue, On spectral disjointness of powers for rank-one transformations and Möbius orthogonality, J. Functional Analysis 266 (2014), 284-317.

[4] H. Abdalaoui, J. Kułaga-Przymus, M. Lemańczyk and T. de la Rue, The Chowla and the Sarnak conjectures from ergodic theory point of view, Discrete Contin. Dyn. Syst. 37 (2017), no. 6, 2899-2944.

[5] T. M. Apostol, Introduction to analytic number theory, Springer-Verlag, New York-Heidelberg, 1976, Undergraduate Texts in Mathematics

[6] F. Bernstein, Über eine Anwendung der Mengenlehre auf ein aus der Theorie der säkularen Störungen herrhrendes Problem, Mathematische Annalen, 71 (3): 417-439

[7] J. Bourgain, On the correlation of the Moebius function with rank-one systems, J. Anal. Math. 120 (2013), 105130.

[8] J. Bourgain, P. Sarnak, T. Ziegler, Disjointness of Moebius from horocycle flows. From Fourier analysis and number theory to radon transforms and geometry, 67-83, Dev. Math., 28, Springer, New York, 2013.

[9] R. Bowen, Topological entropy and axiom A. In Global Analysis (Proc. Sympos. Pure Math. XIV, Berkeley, 1968) (American Mathematical Society, RI, 1970), pp. 23-41

[10] J. Brillhart, P. Morton, A case study in mathematical research: the Golay-Rudin-Shapiro sequence. Amer. Math. Monthly 103 (1996), no. 10, 854-869

[11] H. Davenport, On some infinite series involving arithmetical functions. II, Quart. J. Math., Oxford Ser. 8,(1937), 313-320.

[12] E. Dinaburg, On the relations among various entropy characteristics of dynamical systems. Math. USSR-Izv. 5, 337-378 (1971)

[13] N. Frantzikinakis, Ergodicity of the Liouville system implies the Chowla conjecture, https: //arxiv.org/abs/1611.09338.

[14] B. Green, T. Tao, The Moebius Function is strongly orthogonal to nilsequences, to Ann. of Math. (7) 175, 541-566 (2012).

[15] P. Sarnak, Mobius randomness and dynamics, Not. S. Afr. Math. Soc. 43 (2012), No. 2, 8997.

[16] P. Sarnak, Three lectures on the Mobius Function randomness and dynamics, publications.ias.edu: http://www.math.ias.edu/files/wam/2011/PSMobius.pdf.

[17] P. Sen, J. Singer, Large sample methods in statistics. Chapman \& Hall, Inc. (1993).

[18] D. Karagulyan, On Mobius orthogonality for interval maps of zero entropy and orientationpreserving circle homeomorphisms. Ark. Mat., Volume 53, Issue 2, pp 317-327.

[19] N. M. Korobov, A. G. Postnikov, Some general theorems on the uniform distribution of fractional parts (Russian), Dokl. Akad. Nauk SSSR 84, 217-220 (1952).

[20] I. Kátai, A remark on a theorem of H. Daboussi. Acta Math. Hungar. 47 (1986), no. 1-2, 223-225.

[21] K. Matomäki, M. Radziwiłł, Multiplicative functions in short intervals. Ann. Math. (2) 183(3), 1015-1056 (2016)

[22] K. Matomäki, M. Radziwiłł, T. Tao, An averaged form of Chowla's conjecture, Algebra Number Theory 9 (2015), 2167-2196

[23] T. Tao, The Chowla conjecture and the Sarnak conjecture, https://terrytao.wordpress. com/2012/10/14/the-chowla-conjecture-and-the-sarnak-conjecture/

[24] E. C. Titchmarsh, The theory of the Riemann zeta-function, Second edition. Edited and with a preface by D. R. Heath-Brown. The Clarendon Press, Oxford University Press, New York, 1986.

[25] I. M. Vinogradov, Some theorems concerning the theory of primes, Math. Sb. N. S., 2 (1937), 179-195. 
[26] A. Wintner. Random factorizations and Riemanns hypothesis. Duke Mathematical Journal, 11, no. 2 , pp 267-275. 1944

D. Karagulyan, Department of Mathematics, Royal Institute of Technology, S-100 44 Stockholm, Sweden

E-mail address, D. Karagulyan: davitk@kth.se 